\title{
The effects of sevoflurane and desflurane anesthetics on regional cerebral oxygen saturation during the operation of lower extremities with pneumatic tourniquet
}

\section{Airi KUMASAKA, Ryo AKIMOTO, Asumi SUGIURA, Shinya ODA, Kaneyuki KAWAMAE}

Department of Anesthesiology, School of Medicine, Yamagata University, Yamagata, Japan

\section{Introduction}

Hemodynamic status changes by deflating the pneumatic tourniquet during lower extremity surgery, and it sometimes triggers severe complications for elderly patients ${ }^{1}$.

- As we reported previously, we investigated the effects of sevoflurane and propofol on regional cerebral oxygen saturation $\left(\mathrm{rSO}_{2}\right)$ during the operation of lower extremities with pneumatic tourniquet.

Sevoflurane group indicated significantly higher levels of $\mathrm{rSO}_{2}$ than propofol group. (Fig.1)

It is not certain whether the results are applicable to other inhalator anesthetic agents.

- The goal of this study was to determine whether $\mathrm{rSO}_{2}$ values differ between sevoflurane and desflurane anesthesia during lower extremity surgeries that require pneumatic tourniquet.

\section{Materials and Methods}

- 44 patients undergoing operation of lower legs were included, and randomly divided into sevoflurane (sevo) and desflurane (des) groups.

- Inclusion criteria: The American Society of Anesthesiologists physiological status 1 or 2 patients.

- Exclusion criteria: Under 18 years old patients and/or with past history of cerebral disease.

- Around 1.0 MAC of anesthetic gas was adjusted to control the BIS values within $40-60$, and mechanically ventilated with $\mathrm{FiO}_{2} 0.4$ and maintained normocapnia.

- Physiological parameters, arterial blood gas analysis and $\mathrm{rSO}_{2}$ values were recorded 5 min before, 5, 10 and 15 min after deflation of the tourniquet.

- Patients were followed for a week after the operation to record complications.

- All data were expressed as the mean \pm SD and statistical comparisons were evaluated by Willcoxon rank sum test. $P$-values less than 0.05 were considered as statistically significant.

- $\Delta \mathrm{rSO}_{2}=\mathrm{rSO}_{2}$ values at each time points - baseline $\mathrm{rSO}_{2}$ ( $5 \mathrm{~min}$ before the deflation)

\section{Results}

Table.1 Patient demographic and Clinical characteristics

\begin{tabular}{|c|cc}
\hline & sevo & des \\
\hline $\mathrm{n}$ & 22 & 22 \\
Sex (M/F) & $7 / 15$ & $9 / 12$ \\
Age (Yr) & $68.0 \pm 14.9$ & $76.2 \pm 7.9$ \\
Body weight (kg) & $60.1 \pm 14.8$ & $61 \pm 12.1$ \\
Height (cm) & $154.1 \pm 9.5$ & $151.5 \pm 8.4$ \\
BMI (kg/cm2) & $25.5 \pm 4.5$ & $26.4 \pm 4.6$ \\
Duration of surgery (min) & $132.3 \pm 34.5$ & $120.1 \pm 36.8$ \\
Duration of anesthesia (min) & $204.5 \pm 39.2$ & $185.9 \pm 41.6$ \\
Duration of tourniquet time & $89.9 \pm 24.8$ & $88.4 \pm 27.3$ \\
(min) & $16.0 \pm 50.4$ & $17.6 \pm 74.9$ \\
Estimated blood loss (ml) & $218.0 \pm 196.4$ & $171.4 \pm 139.0$ \\
Urine output (ml) & $1204.5 \pm 380.0$ & $1077.3 \pm 359.2$ \\
Intake (ml) & 0.0 & $20.5 \pm 93.7$ \\
MAP (ml) & $2.0 \pm 0.7$ & $1.8 \pm 0.7$ \\
Total dose of Remifentanil & & \\
(mg) & $2.6 \pm 4.2$ & $2.7 \pm 3.9$ \\
use of ephedrine (mg) & *Statistically different among the groups
\end{tabular}

Table.2 Blood gas analysis and hemodynamics

\begin{tabular}{|c|c|c|c|c|c|c|c|c|}
\hline & sevo & des & sevo & des & sevo & des & sevo & des \\
\hline & \multicolumn{2}{|c|}{ 5min before deflation } & \multicolumn{2}{|c|}{ 5min after deflation } & \multicolumn{2}{|c|}{ 10min after deflation } & \multicolumn{2}{|c|}{ 15min after deflation } \\
\hline $\begin{array}{c}\text { MAP } \\
(\mathrm{mmHg})\end{array}$ & $70.1 \pm 10.1$ & $73.2 \pm 12.6$ & $56.8 \pm 8.5$ & $57.5 \pm 9.2$ & $56.2 \pm 7.8$ & $58.2 \pm 6.6$ & $58.7 \pm 9.0$ & $56.6 \pm 6$ \\
\hline $\begin{array}{c}\text { HR } \\
\text { (bpm/m) }\end{array}$ & $60.6 \pm 6.9$ & $60.5 \pm 8.9$ & $60.0 \pm 8.0$ & $61.7 \pm 8.8$ & $61.0 \pm 7.7$ & $63.9 \pm 9.3$ & $61.1 \pm 7.5$ & $65.0 \pm 7.9$ \\
\hline BIS & $45.4 \pm 6.5$ & $43.2 \pm 8$ & $47.0 \pm 8.2$ & $44.6 \pm 6.7$ & $47.2 \pm 7.6$ & $46.1 \pm 7.1$ & $47.4 \pm 7.1$ & $46.5 \pm 7.8$ \\
\hline $\mathrm{Hb}(g / d \mathrm{l})$ & $11.4 \pm 1.5$ & $11.6 \pm 1.7$ & $11.5 \pm 1.5$ & $11.5 \pm 1.9$ & $11.2 \pm 1.5$ & $11.4 \pm 1.7$ & $11.1 \pm 1.6$ & $11.3 \pm 1.7$ \\
\hline $\begin{array}{c}\mathrm{EtCO}_{2} \\
(\mathrm{mmHg})\end{array}$ & $36.6 \pm 2.8$ & $37.4 \pm 2.6$ & $40.0 \pm 4.4$ & $41.7 \pm 3.6$ & $39.0 \pm 4.2$ & $41.3 \pm 3.1$ & $38.3 \pm 4$ & $40.7 \pm 3.2$ \\
\hline $\begin{array}{c}\mathrm{PaCO}_{2} \\
(\mathrm{mmHg})\end{array}$ & $38.4 \pm 4.4$ & $37.6 \pm 3.3$ & $43.1 \pm 3.9$ & $42.3 \pm 3.3$ & $41.8 \pm 4.7$ & $41.5 \pm 3.4$ & $40.7 \pm 3.7$ & $41.2 \pm 3.2$ \\
\hline $\begin{array}{c}\mathrm{PaO}_{2} \\
(\mathrm{mmHg})\end{array}$ & $155.3 \pm 39.6$ & $131.1 \pm 28.5$ & $169.5 \pm 31.5$ & $135.7 \pm 26.5$ & $171.9 \pm 35.0$ & $141.6 \pm 28.5$ & $169.0 \pm 35.3$ & $139.5 \pm 31.0$ \\
\hline P/F ratio & $365.2 \pm 93.4$ & $337.9 \pm 70.1$ & $339.2 \pm 77.7$ & $352.4 \pm 65.1$ & $404.9 \pm 85.7$ & $365.4 \pm 71.1$ & $317.9 \pm 86.2$ & $359.6 \pm 76.6$ \\
\hline $\begin{array}{l}\text { Et anes } \\
\text { gas con }\end{array}$ & $1.3 \pm 0.2$ & $3.8 \pm 0.5$ & $1.2 \pm 0.2$ & $3.6 \pm 0.5$ & $1.2 \pm 0.2$ & $3.5 \pm 0.5$ & $1.1 \pm 0.2$ & $3.4 \pm 0.6$ \\
\hline $\begin{array}{c}\mathrm{Cl} \\
\left(\mathrm{L} / \mathrm{min} / \mathrm{m}^{2}\right)\end{array}$ & $2.3 \pm 0.9$ & $2.5 \pm 0.5$ & $2.4 \pm 0.8$ & $2.7 \pm 0.8$ & $2.6 \pm 0.9$ & $2.9 \pm 0.9$ & $2.5 \pm 0.8$ & $2.9 \pm 0.7$ \\
\hline $\mathrm{rSO}_{2}(\%)$ & $64 \pm 10.1$ & $66.8 \pm 6.7$ & $64.4 \pm 9.4$ & $66.0 \pm 5.6$ & $\begin{array}{r}65.5 \pm 8.6 \\
* S t a\end{array}$ & $\begin{array}{c}67.8 \pm 5.6 \\
\text { tically diffe }\end{array}$ & $\begin{array}{l}65.7 \pm 8.5 \\
\text { nt among th }\end{array}$ & $\begin{array}{l}68.1 \pm 5.9 \\
\text { groups }\end{array}$ \\
\hline
\end{tabular}

- There was significant differences between the groups in the age of patients. But we confirmed the age of patients was not significantly affected to the $\mathrm{rSO}_{2}$ values by regression analysis.

- Sevo group had significantly higher $\mathrm{rSO}_{2}$ values than desflurane group 5 minutes after deflation. $(p=0.03)$

- Sevo group indicated continuous increase in $\mathrm{rSO}_{2}$ values after deflation.

- However, des group showed decline in $\mathrm{rSO}_{2}$ values after deflation.

- There were no significant differences between the groups in terms of post-operative complications.

\section{Discussion and Conclusion}

Fig.2 $\quad \mathrm{rSO}_{2}$ Sevoflurane vs. Desflurane

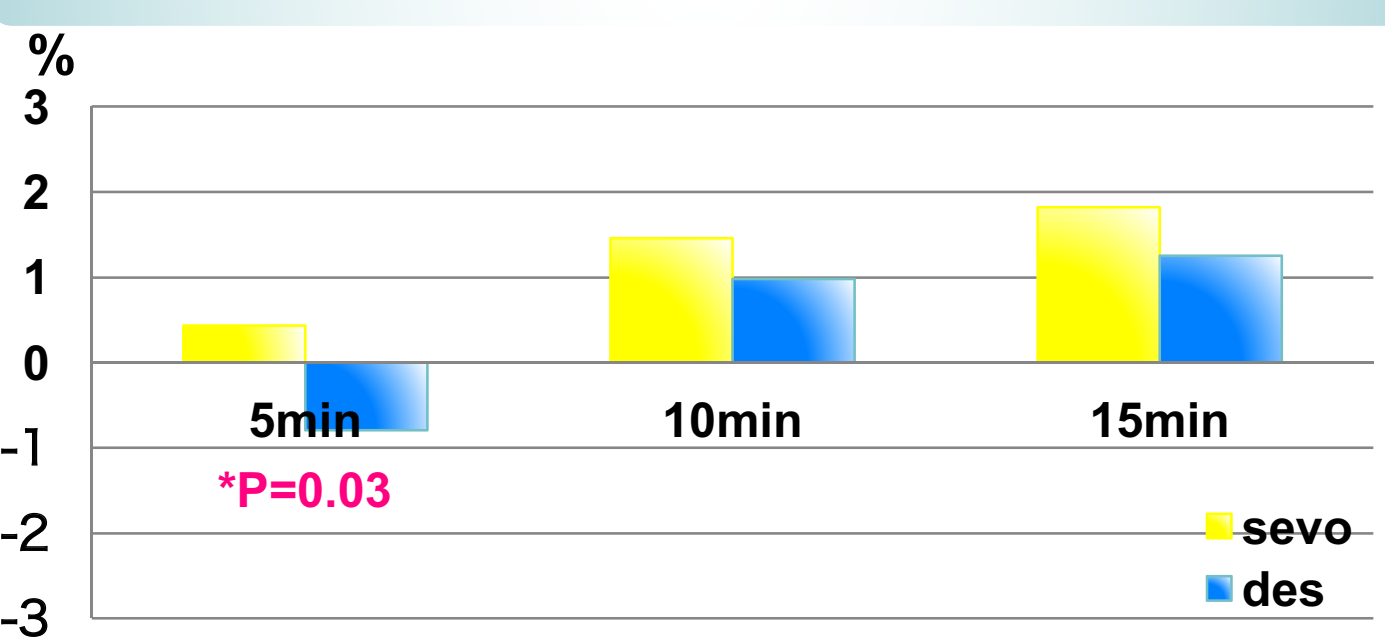

- 5 minutes after deflation of tourniquet, there was significant difference between two inhalational anesthetic agents on $\mathrm{rSO}_{2}$.

- It is known inhalational anesthetics impair cerebral autoregulation, but only the sevoflurane can preserve the cerebral autoregulation at the concentration of clinical use ${ }^{2}$.

- And some chemical mediators might to this results. For instance, sevoflurane is known to induce the Hypoxia Inducible Factor, and it has cytoprotective effects on ischemic period ${ }^{3}$.

\section{References}

1) Wakai A et al, J Am Acad Orthop Surg 2001; 9: 345-51

2) Summors et al, Anesth Analg 1999; 88: 341-345

3) Zitta et al, Eur. J. Pharmacol 2010; 645: 39-46 